\title{
The use of fluorescence-activated cell sorting in studying plant development and environmental responses
}

\author{
ANTHONY D. CARTER ${ }^{1,2}$, ROXANNA BONYADI ${ }^{1}$ and MIRIAM L. GIFFORD*,1,2 \\ ${ }^{1}$ School of Life Sciences and ${ }^{2}$ Warwick Systems Biology Centre, University of Warwick, Coventry, UK
}

\begin{abstract}
Fluorescence-Activated Cell Sorting (FACS) is a powerful tool that enables plant growth and development to be studied at the cellular level. Flow cytometry is used to isolate subpopulations of cells, such as those of specific cell types, or cells at particular developmental stages that have been marked with fluorescent proteins. Transgenic technology has given us the ability to generate plants that express fluorescent proteins, not just constitutively in particular cell types, but also dynamically in response to endogenous or external factors. By processing such transgenic lines with FACS, it is possible to isolate distinct populations of cells in a wide range of likely response states for further analysis. This is particularly useful for investigating biological mechanisms in plants because the control of growth and development is manifest at the cell type level. Furthermore, the specificity of the resulting data enables fine modelling of the transcriptional networks that exert systems-level control of the transcriptome; hence key regulators of responses and processes in the plant can be identified. In this review, the current state of the art for FACS methods in plants is explored by means of case studies of research in which cell sorting allowed us to make significant new discoveries.
\end{abstract}

KEY WORDS: cell sorting, cell specificity, plant root, fluorescent reporter, Arabidopsis

\section{Introduction}

Multicellular organisms, such as plants rely on highly specialised cell types to perform discrete functions. For example, in the root, gravity is sensed in columella cells and reactivation of cell division in differentiated pericycle cells results in formation of lateral root primordia. In the leaf, stomatal guard cells play a critical role in regulating gaseous exchange between the plant and its environment, while carbon fixation occurs in mesophyll cells. To add a further layer of complexity, different cells within a single organ could be dividing, elongating, or differentiating; active, stressed, or dead; and/or responding to stimuli in subsets of their cellular type. Technologies that allow us to determine the genetic components that lead specialised cells to acquire specialised functions, one of the hallmarks of multicellular organisms, facilitate otherwise elusive insights into plant growth and development.

The consideration of cell specificity is intrinsic in the study of plant developmental biology and at the heart of research aiming to understand cell identity and developmental plasticity. Cell ablation, for example, is a tool that has been used to understand how the position of a cell relative to its neighbours influences cell fate (Kidner et al., 2000). At the genetic level, fluorescent tagging of promoters or genes has been employed to investigate cell-cell signalling events (Nakajima et al., 2001) and used in many studies to express genes in subsets of cells to mimic in vivo gene functions (Liu et al., 2012). To complement functional genetic studies, gene expression profiling has become an important tool for describing both the scope of biological phenomena and identifying candidate genes involved in particular functions. Problematically, however, such genomic studies tend to ignore the importance of cell specificity and analyse the transcriptome of whole organs. This is a critical limit for interpreting transcriptomic data, since signal cascades from distinct cells could be confounded because samples are comprised of mixed cell types, each with individual levels and states of gene expression.

More recently, a number of technologies have been applied to allow genomic analysis of particular cellular molecules at the cell-type- or response-stage-specific level. The complete transcriptome can be studied using either green fluorescent protein (GFP)-expressing plants for protoplast generation and subsequent

Abbreviations used in this paper: GFP, green fluorescent protein; FACS, fluorescenceactivated cell sorting.

\footnotetext{
*Address correspondence to: Miriam L. Gifford, School of Life Sciences, University of Warwick, Gibbet Hill Road, Coventry CV4 7AL, UK. Tel: +44-2476-575-268. Fax: +44-2476-574-500. e-mail: miriam.gifford@warwick.ac.uk
} 
fluorescence-activated cell sorting (FACS) (Birnbaum et al., 2003) or laser-capture microdissection (Kleber and Kehr, 2006). The active transcriptome can be studied by means of the Isolation of Nuclei TAgged in specific Cell Types (INTACT) system for nuclear precipitation (Deal and Henikoff, 2010) and the translatome by immunoprecipitation of polysomes (Mustroph et al., 2009). Each of these technologies has advantages and disadvantages (for a review, see Rogers et al., 2012). This review discusses FACS, one of the most cost- and time-effective procedures for obtaining samples of great enough cell quantity and quality to facilitate accurate investigation of cellular 'omics'.

\section{Plant development is mediated by cell-type specific processes}

A fundamental aspect of multicellularity is the range and scale of cell differentiation. This differentiation is observable from the very first stages of plant development. For example, the Arabidopsis thaliana zygote divides asymmetrically into two daughter cells with distinct cell fates. The small apical cell gives rise to most of the embryo, whereas the larger basal daughter cell forms mainly the extra embryonic suspensor (Laux et al., 2004). Differences between cell types compared to each other and also the range of developmental stages in which a single cell type can exist, are both key aspects of cell specificity in the embryonic, juvenile, and mature plant. This enables each cell type to take on particular roles and functions, underpinned by the distinct transcriptomes expressed by each cell type or state. Cell type identity is also involved in determining which cells are capable of sensing internal and external conditions. For example, epidermal cells form the frontier between plant root or shoot and environment, and are responsible for recognising exogenous signals, such as pathogens and the availability of nutrients. Furthermore, the root epidermal trichoblast cells - root hairs - are one of the key sites for nutrient uptake in all plants while also playing a key role in the early stages of nodule formation in legumes. In the leaf, the formation of stomata through the differentiation of guard cells is vital for transpiration and gas exchange between the plant and the environment (for a recent review, see Lau and Bergmann, 2012). As such, the function and proliferation of guard cells is of particular relevance for improving water use efficiency (Davies et al., 2002).

Aside from mediating growth and nutritional uptake, cell-specific programs are fundamental to the developmental organisation of plant architecture. For example, lateral root organogenesis is initiated specifically in the pericycle through a program of asymmetric, anticlinal cell division (Malamy and Benfey, 1997). A similar cell-type re-initiation of cell division is observed in the early stages of nodule formation in leguminous plants. During nodulation, Nod factor signalling in the epidermis is followed by protraction of an infection thread through root hairs into the cortex. At the same time, there is very rapid reactivation of cell division in cortical cells several layers below, to form the meristem of the developing nodule (reviewed in Oldroyd and Downie, 2008). While knowledge of the interlinking of plant response pathways to rhizobia and mycorrhiza is well established (e.g., Hirsch and Kapulnik, 1998; Geurts et al., 2005), the mechanistic connections between lateral root development and nodule formation are less well studied, but are evident at the cell-specific level.

Cell specificity mediates cellular responses to the environment and their plasticity. Understanding the scale of plasticity is highly important in the area of developmental biology, but can also help apply research to modify the efficiency by which plants use nutrients or water, or grow and develop. For example, the opening of stomata is regulated by the time of day and $\mathrm{CO}_{2}$ levels (Morison, 2006); hence, altering the response to such stimuli in those specific cells at particular times of day could reduce water loss by transpiration.

\section{FACS is a high-throughput, accurate method for isolating cell types that preserves the endogenous transcriptome}

FACS is a type of flow cytometry technique that allows a mixed population of cells to be analysed and sorted based on fluorescent properties (Fig. 1). The technique and technology (Bonner et al., 1972) was developed initially to isolate mouse (Mus musculus) spleen cells bound to lactose-keyhole limpet haemocyanin (immunofluorescently labelled to allow sorting) by investigating the antigen productivity of cells derived from antigen-binding cells (Julius et al., 1972). FACS became a commonly used method for sorting cell populations that may be easily separated from each other, such as hematopoietic or cultured cells (Nelson, 1999; Mattanovich and Borth, 2006). However, a decade ago, a number of methods and developments enabled FACS of plant cells.

The first key development to cell sorting in plants was the increasing availability of transgenic plants expressing fluorophores in subsets of cell types. Any fluorophore can be used to mark cells for FACS, although in plants the use of GFP predominates due to the availability of GFPlines. These include both promoter:GFPlines that tag individual gene promoters (such as the pSCARECROW::GFP root endodermal marker; Birnbaum et al., 2003) and large enhancer trap collections in which a construct expressing the GAL4 DNAbinding domain, the Upstream Activating Sequence, and GFP enables fluorescent expression under the control of regulatory regions at or near the insertion site (Haseloff, 1999), such as GFP marking lateral root founder cells in E3754 (Gifford et al., 2008). The second key component for facilitating FACS in plant cells was to deal with the presence of the protective cell wall. Methods were developed for protoplast generation by using cell wall-degrading hydrolytic enzymes to dissociate plant root cells (Birnbaum et al., 2005). Thirdly, a adaptation was needed to be able to employ genome-level analysis to the small amounts of material that could be obtained from the rare cell types isolated. Unbiased amplification of RNA is now available thanks to kits (from a number of commercial suppliers) and advances continue in the molecular biology for transcriptomics direct from pico quantities of RNA.

The first uses of FACS combined with whole-genome analysis using microarrays in plants aimed to dissect transcription in the plant root (Birnbaum et al., 2003; Brady et al., 2007). Analysis of the resulting data revealed novel cell-specific expression patterns that were masked in parallel analyses of the whole root. The unmasking showed gene expression that was either highly cell-type-specific or that exhibited a mixed induction/repression pattern across cell types, thus highlighting the power of FACS for identifying complex expression patterns. A range of techniques, including in situ hybridisation, were utilized to evaluate the 'digital in situ' FACS expression pattern to validate the microarray data and confirm that FACS samples reflected endogenous expression patterns. FACS was found to preserve the majority of endogenous responses in spite of the protoplast generation procedure. The small number 
of genes that are significantly affected by protoplast generation or FACS can be removed from subsequent analysis without major loss of genomic detail (Birnbaum et al., 2003; Gifford et al., 2008).

\section{Understanding gene expression across cell types and developmental zones in the Arabidopsis root}

A key aim of cell-type-specific analysis in any multicellular organism is to map molecular components across each cell type. Birnbaum and colleagues (2003) were the first to chart relative gene expression across different cell types of the Arabidopsis root (Birnbaum et al., 2003). Root cell types were GFP-marked and sorted in five populations - stele, endodermis, endodermis + cortex, epidermis, and the lateral root cap. In addition, dissection was used to obtain samples from three different developmental stages -the root tip/meristematic zone, the elongation zone, and the zone where cells are fully elongated/differentiating. Dissection and FACS were combined to generate 15 root zone samples. A k-

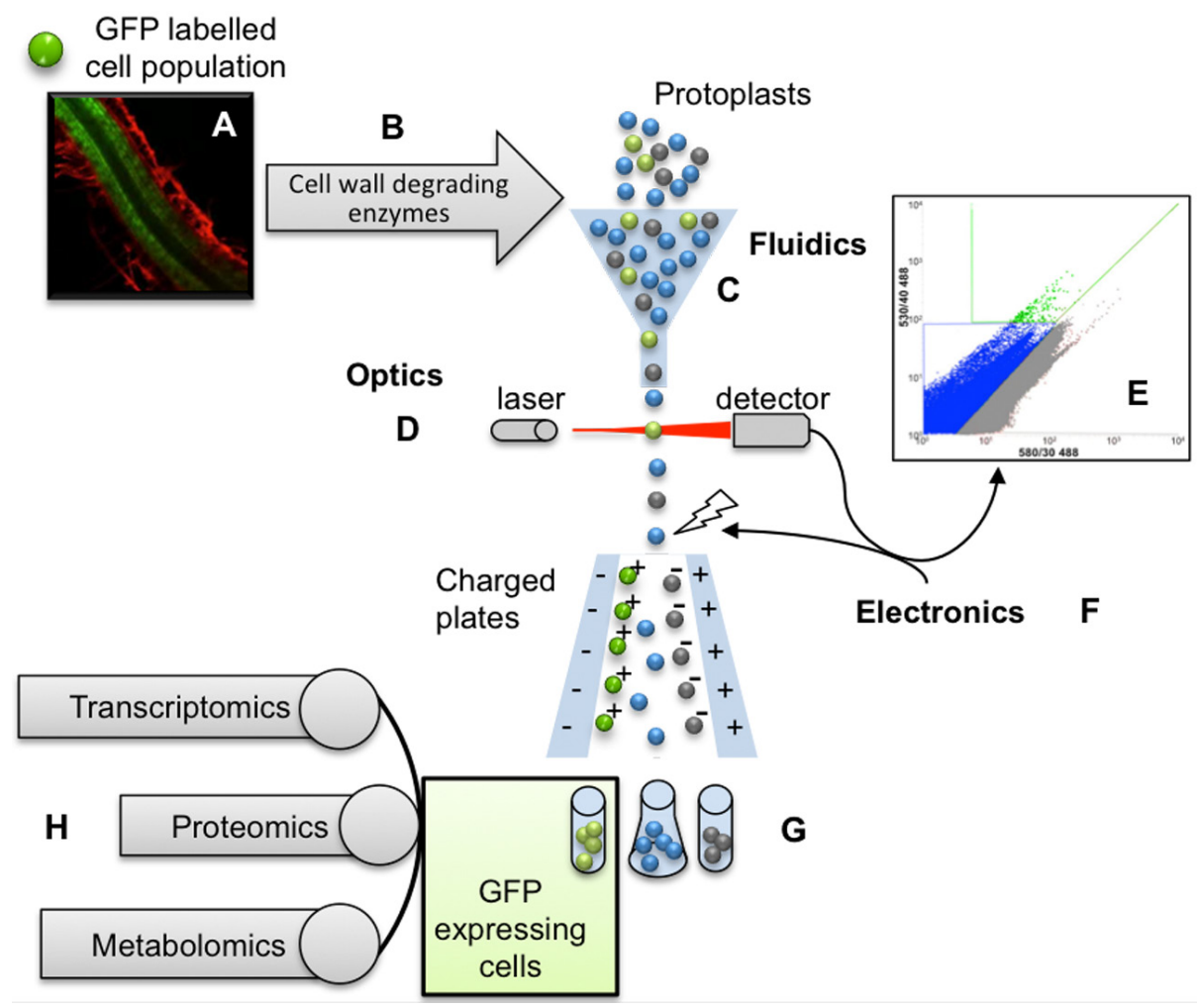

Fig. 1. Fluorescence-Activated Cell Sorting (FACS) workflow. (A) Transgenic plants carrying a fluorescent reporter construct conferring cell-or tissue-specific expression are chosen for an experiment; for instance, Medicago truncatula roots expressing GFP expressed in the cortex to investigate nodule development in cortical cells. (B) Roots are harvested and treated with enzymes to dissociate cells, which are then filtered to break up large cell clumps. (C) In a typical FACS machine, the cell sample is injected into a sheath fluid sort stream, then the stream is vibrated at high frequency to break it into uniform droplets containing no more than one cell each. (D) Laser light and detection filters are combined to measure the fluorescence and other properties (such as size) of each droplet. (E) The emission spectrum is analysed and appropriate 'gates' of wavelength determined to define the positive and negative droplets for sorting. (F) An electrical charge is imparted on droplets within each gate. (G) Electrical plates deflect charged droplets (here illustrated for two-way sorting (green/ grey shaded cells) with a waste collection of all other cells (blue)l. (H) Cells are collected into tubes and then used for visual analysis (for instance, confirmation of fluorescence levels) or collected into tubes that contain buffer to immediately lyse cells for rapid molecular extraction. means clustering of the zone-specific expression data was used to a map of localised gene expression within the root, identifying ter Fig. 2A) (Birnbaum et al., 2003).

A total of 10,492 genes were determined to be expressed in at least one zone, of which 5,712 genes were found to vary in striking level of zonal specificity and it highlights the heterogeneity of expression within a plant organ, validating the use of cell-specific pis to accurately analyse plant development. The study also implicated specific genes in controlling cell specificity since the exhibit specificity to particular LEDs. For example, of developmental control, metabolism, and stress response (Martin In the lateral root cap, WRKY TFs, genes known to perform key signalling roles across a range of plant processes (Rushton et al., 2010) were prevalent (Fig. 2A).

Within the Arabidopsis root, there are several very low abundance cell types, including the four cell quiescent centres (QCs). The specificity of FACS was suitable to profile the QC. By comparison with the data of Birnbaum et al., (2003), Nawy et al., (2005) were able to identify 290 QC-enriched transcripts (Fig. 2B). Functional analysis was used to explore the QC-enriched genes, uncovering a high level of redundancy, perhaps an indication of the critical importance of this cell type (Nawy et al., 2005).

Following the early plant FACS work, the expression map concept was taken further using greater longitudinal resolution and coverage of almost all root cell types (Brady et al., 2007). Eight new GFP-marked lines were sorted and combined with existing FACS data to collectively form a 19 cell population dataset, and samples were taken from 13 longitudinal 5-cell-long root sections extending from the columella to the maturation zone. Studying increased numbers of cell types delivered the resolution to identify genes with expression peaks in multiple cell types. The vast longitudinal resolution of this study also allowed investigation into temporal variation in gene expression, with each longitudinal section representing a developmental timepoint. A number of genes were found to exhibit fluctuating expression levels along the longitudinal axis, surprising given the expectation that cells close together would exhibit more similar expression patterns compared with those further apart. However, this may be seen comparable to the priming of only a select number of pericycle cells for the later reactivation of cell division and differentiation 
into lateral root primordia (De Smet et al., 2007). To investigate this developmental specificity, FACS was used to profile the pericycle cells that reactivate cell division by analysing xylem-pole pericycle cells exposed to inductive auxin treatment over a 6-hour time series (De Smet et al., 2008). The 1,920 differentially expressed genes implicated in the early stages of lateral root organogenesis from the FACS transcriptome, could be narrowed down to one gene, ARABIDOPSIS CRINKLY4 (ACR4) -known to function in cell layer organisation in other tissues (Gifford et al., 2003) - that was identified as a new key regulator of formative pericycle cell division (Fig. 2D).

\section{Zonal specificity of gene expression in the shoot apical meristem}

FACS methods for plants were first developed in roots and have improved our understanding of roots considerably. The plant shoot is composed of as equally complex a set of cell types, but FACS is made more challenging due to chlorophyll autofluorescence of leaf cells. Nevertheless, more recently, methods have been developed, to isolate, for instance, guard cells in the leaf (Grønlund et al., 2012), and the shoot apical meristem (SAM) has been isolated and the transcriptome profiled (Yadav et al., 2009). Due to the wild-type
A

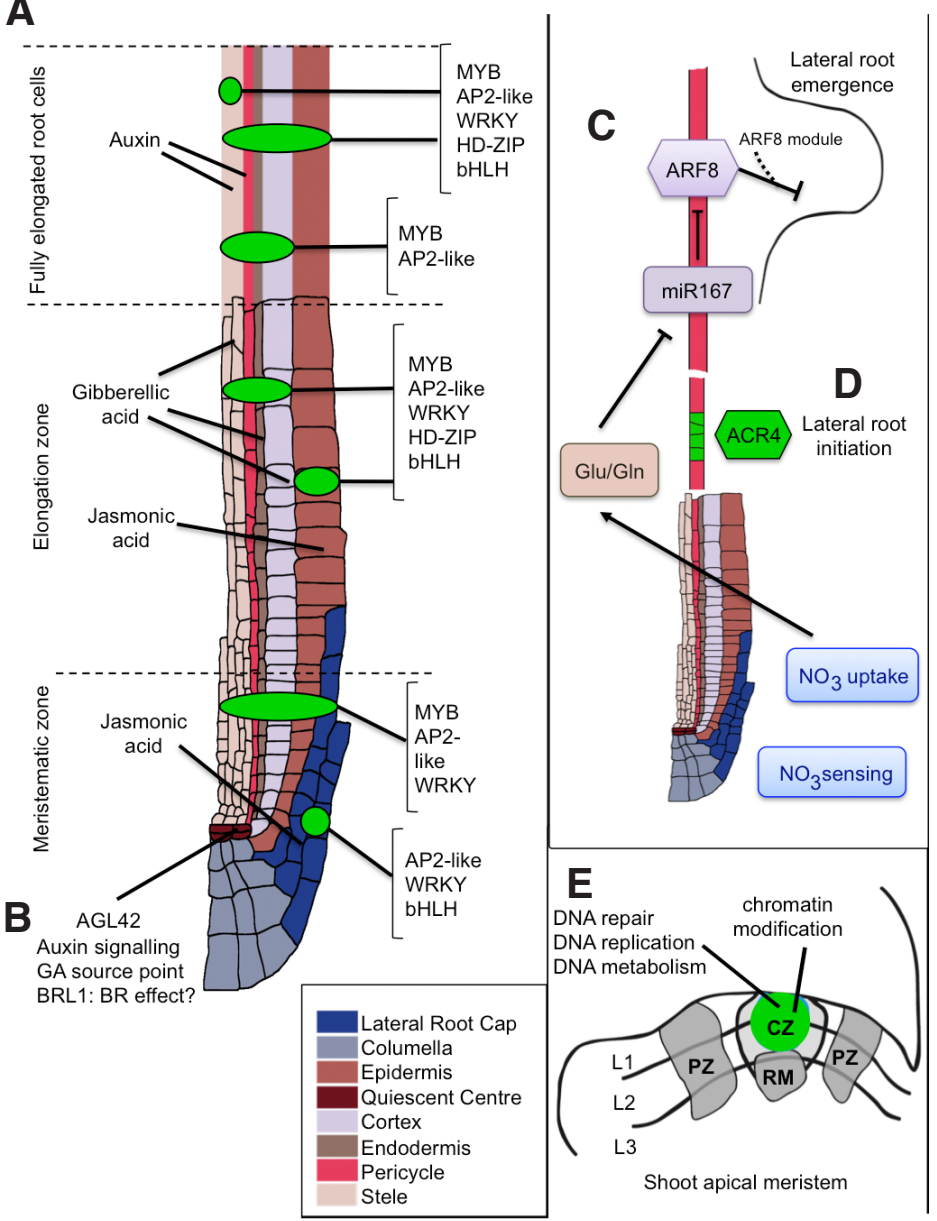

$\mathbf{F}$

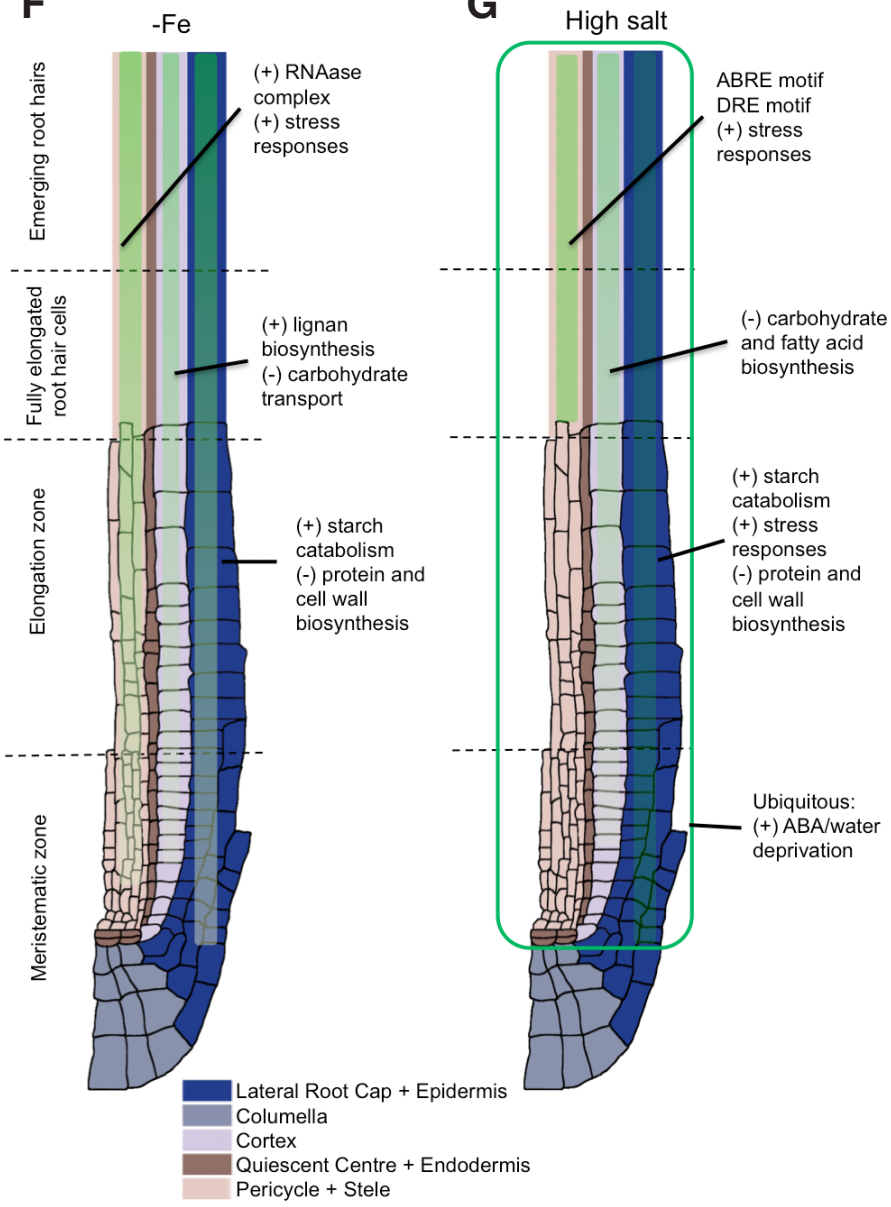

Fig. 2. Novel discoveries from fluorescence-activated cell sorting (FACS) enabling better understanding of plant growth and development. (A) Cell-type and developmental stage-specific results from Birnbaum et al., (2003). Phytohormones (left, arrows pointing to the cell type within each stage) and transcription factor families (right, green highlights indicating the cell types within each stage) together control root development by mediating cell-type-specific functions or responses. (B) Analysis of expression in AGAMOUS-LIKE42 (AGL42)-marked QC cells compared to other root cell types in Nawy et al., (2005) identified 290 QC-enriched transcripts. The functions of the 290 QC genes suggest that the QC acts as a hormone signalling centre. (C) FACS used to identify a novel pericycle-specific nitrogen-regulated control of lateral root development (Gifford et al., 2008). Assimilated nitrate $\left(\mathrm{NO}_{3}\right)$ in the form of glutamate/glutamine (G/u/GIn) represses microRNA167, in turn repressing ARF8, which controls a regulatory module that induces lateral root primordia development but represses primordium emergence. (D) The first cell divisions of lateral root development in the pericycle were found to be controlled by the receptor-like kinase ACR4 (De Smet et al., 2008) by means of transcript profiling of auxin-activated FACS-isolated xylem pole pericycle cells. (E) Separating the Arabidopsis SAM into three cell regions - peripheral zone (PZ), rib meristem (RM), and central zone (CZ) - by FACS enabled identification of stem-cell-enriched processes (Yadav et al., 2009). (F,G) Responses to iron-depletion (F) and high salt conditions (G) were FACS-profiled across four developmental zones and in six cell types (Dinneny et al., 2008). This analysis of how root cell types coordinate stress revealed that more ubiquitous responses are stress specific, whereas cell-type-specific biological processes are common targets for stress regulation, such as cell wall biosynthesis, consistent with phenotypic effects. An overview of some of the processes identified and whether they are positively (+) or negatively (-) affected by the stress is shown according to cell/stage location (green shading, with the less diffuse green for stronger localisation). 
Fig. 3. Analysis of the magnitude and cell specificity of gene expression in four Arabidopsis root transcriptome studies. Density distributions of maximum fold changes between cells (calculated using the density function in $R$, with $n=10,000 ; R$ Development Core Team, 2012) belonging to cell types isolated by FACS (black) and developmental stages isolated by dissecting roots at intervals (red): (A) Expression map of five radial cell types and three longitudinal zones (Birnbaum et al., 2003). Similar maximal fold changes occur between cell types and developmental stages, with only a slightly higher density around a fold change of 3 , indicating a marginally greater variation between cell types. (B) Expression map of 19 radial cell types and 13 longitudinal zones (Brady et al., 2007). Distributions highlight a high degree of cell-specific gene expression. The increased longitudinal resolution of the dataset compared to that of Birnbaum et al., (2003) does not alter the distribution of developmental stage densities greatly, with a small increase in peak from 1.42 (A) to 1.97 (B). The peak maximal fold change across cell types is 4.45 , indicating that a large number of observed genes vary greatly within root tissue. (C) Nitrate treatment vs. control in five cell types (Gifford et al., 2008). (D) The nitrate treatment caused a slight increase in frequency of maxima between 3 and 5 compared with controls indicating that nitrate-

responsive genes were highly cell-type specific. (D) Salt treatment and iron deficiency vs. control in six cell types (Dinneny et al., 2008). Similarly, marginal shifts indicating cell type specific responses are observed in both salt treatment and iron deficiency data compared with respective controls.
A

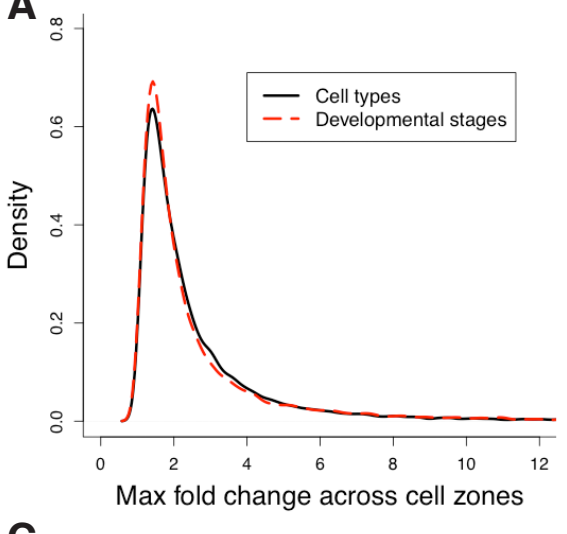

C

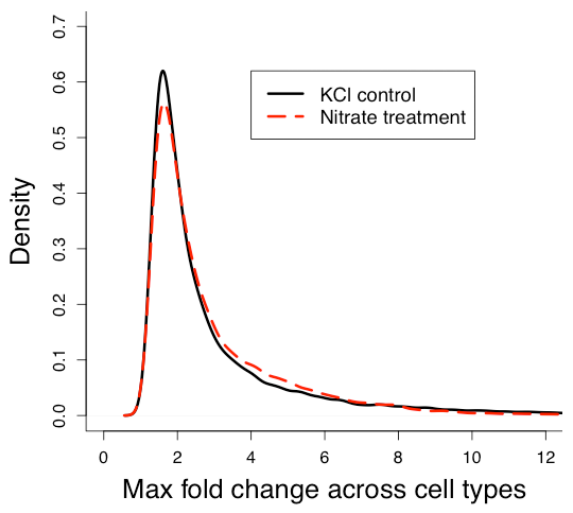

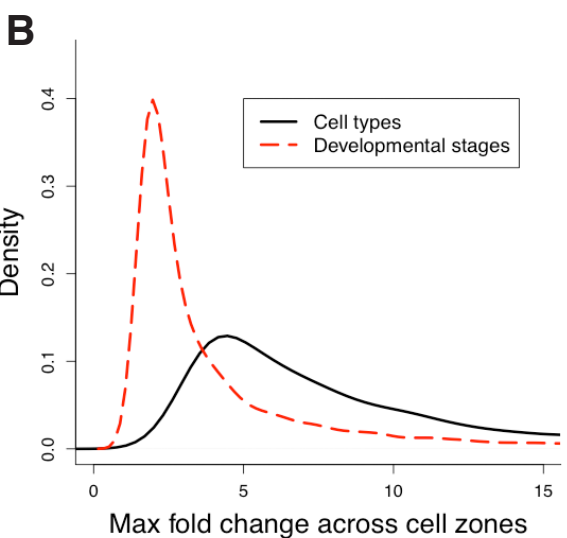

D

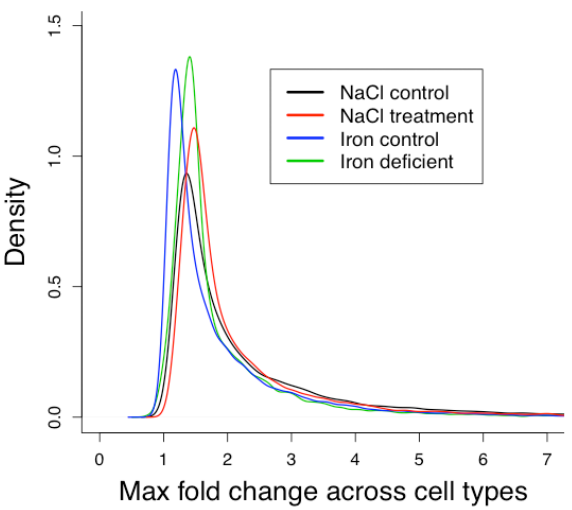

Arabidopsis SAM consisting of only approximately 35 cells of four cell types, Yadav et al., carried out FACS on the double mutant, apetala1-1;cauliflower1-1 (ap1-1;cal1-1), which has an enlarged SAM (Ferrándiz et al., 2000). Three cell zones were labelled with cell-specific promoters driving fluorescent protein expression - the central zone, rib-meristem, and peripheral zone - and the remaining SAM cells obtained by sorting non-GFP-labelled SAM cells (Fig. 2E). FACS-identified expression patterns, verified by in situ RNA analysis, proved a much more sensitive analysis tool than whole shoot/leaf studies. The cell-specific approach showed that the central zone was enriched for genes involved in DNA metabolism, replication, and repair as well as epigenetic regulation, providing new evidence of the similarity to mammalian stem cells that express similar processes.

\section{Arabidopsis roots exhibit a high degree of cell-type specificity in response to abiotic stimuli}

Once it was confirmed that cell sorting preserves the vast majority of endogenous expression levels, FACS techniques were applied to study rapid and transient signalling of environmental factors in the root (Dinneny et al., 2008; Gifford et al., 2008). Celltype specific nitrate responses were analysed by profiling gene expression changes following nitrate influx in five GFP-marked cell type combinations (Gifford et al., 2008). Whole root studies had previously identified 4,139 nitrogen-responsive genes, but in the cell-type-specific study, 6,202 such genes were found. The sensitivity of the FACS profiling allowed detection of nitrogenresponsive transcriptomic reprogramming specific to particular cell types. A novel finding was the control of regulation of the balance between lateral root primordia formation and outgrowth by the AUXIN RESPONSE FACTOR 8 (ARF8) transcription factor via glutamine/glutamate (assimilated nitrate)-repression of miR167 (Gifford et al., 2008) (Fig. 2C). Without the specificity conferred by FACS, this discovery would not have been possible. Comparisons between sorted cell types and whole organs validate the use of FACS, but are also informative: 2,868 of the genes differentially expressed to nitrogen in roots are not identified in the sorted cell populations, but, as the GFP lines used (Gifford et al., 2008) do not encompass all root cell types or developmental stages, this argues for further FACS profiling.

The effects of salt stress and iron deficiency on the root were analysed with three complementary experiments (Dinneny et al., 2008). A time series in whole roots was performed, the responses across longitudinal root sections were obtained via dissection, and FACS was used to isolate individual radial root zones (Fig. 2 F-G). For iron deficiency, ten times as many genes could be identified that respond in isolated cell types compared to longitudinal sections. A large number of genes were uncovered by the time series analysis, but only $10 \%$ of them were found to respond in common with single cell types, suggesting that there is a global time-dependent iron deficiency response, but also a high degree of radial specificity, perhaps indicative of local stress conditions. Salt stress responses were particularly cell type specific, with 
3,862 salt-responsive genes across cell types compared to 569 in whole roots and 1,173 across longitudinal zones. The overlap of just 174 genes between the three experiments suggests that both zonal and cellular responses are distinct and non-uniform. Water-stress-related genes were found to be activated across all cell types as would be expected under high salt conditions, though known salt stress response genes were enriched only in the stele, further indicating the cell-type specificity of root responses to stimuli.

\section{Quantification of the scale of gene expression changes revealed using FACS}

To investigate the level of variation between cell types relative to developmental stages and the effect of abiotic stresses on whole roots, distributions of maximum fold changes between cell zones were analysed. We analysed normalised transcriptome data from Birnbaum et al., (2003) (Fig. 3A) and Brady et al., (2007) (Fig. $3 B$ ) that mapped cell-specific gene expression across cell types and developmental zones, as well as our data investigating celltype-specific responses to nitrogen influx (Gifford et al., 2008) (Fig. 3C) and cell-type-specific responses to salt stress and iron deficiency from Dinneny et al., (2008) (Fig. 3D). In each case, the maximum fold change between cell types/zones were calculated for each gene. The results show that there is an increased level of variability in gene expression between individual cell types compared to the different zones of the root, highlighting the power of FACS in identifying dramatic, yet highly, cell-type-restricted changes in transcript expression (Fig. 3 A-B). Within studies that profiled gene expression changes to treatments in different cell types, FACS identified treatment-dependent responses in cellular patterns and allowed identification of exclusive high magnitude responses specific to single cell types (Fig. 3 C-D).

\section{Cell sorting combined with other technologies}

The ability to carry out FACS in plants has yielded a number of discoveries that were only made possible through the use of cell sorting (Fig. 3), and cell-specific analysis is not limited to simply creating expression maps of organs. FACS can be easily combined with functional genetic analysis, for example, by crossing GFP lines with mutant lines carrying T-DNAs (Scholl et al., 2000). The analysis of the cell-specific effects of mutations on gene expression allows a comparison of the cellular pattern of perturbations, which is highly relevant for interpreting molecular phenotypes controlling development and also for understanding genetic redundancy. For rapid molecular characterisation, protoplast cell cultures are often transformed with overexpression or RNA interference constructs. By incorporating a fluorescent marker in these constructs, it is possible to filter out the cells that were not successfully transfected, culminating in a more homogenous population of cells for analysis of gene perturbation (Bargmann and Birnbaum, 2009).

Cell sorting can refine a number of other molecular methods. Even for cell types that are more easily dissociated, such as male gametophyte cells (pollen), the use of FACS can improve the purity of samples for subsequent analysis (Becker et al., 2003). Furthermore, between each stage of development, pollen shows remarkable levels of stage-specific transcription. This information can be used to select pollen-stage-specific fluorescent markers for subsequent FACS.
Individual cell types are dynamic, comprising cells at different developmental stages and undergoing different responses, and can be separated using combinations of distinguishable fluorescent reporters to enable four-way, two-colour sorting. A prescient example of developmental specificity within a cell type is lateral root formation. The initial stages of lateral root development, from the re-initiation of cell division to the protrusion of the lateral root tip through the epidermis, take place over a short time scale. Put together with the fact that only a small number of pericycle cells initiate lateral root organogenesis (lateral root founder cells), this represents a narrow developmental and spatial window of cells to isolate for an analysis of lateral root development. As discussed above, FACS analysis of this small subset of pericycle cells has been used successfully to identify a new key regulator of lateral root development (De Smet et al., 2008).

\section{Single cell-type to single cell}

FACS is now a widely used technique in biology for analysis of genomic and genetic expression patterns (reviewed in Galbraith, 2012). Two of the new frontiers for single cell-type analysis are further examination of the molecular landscape (metabolome, proteome, etc.) in cell types or refinement of the single cell level. Whereas RNA amplification protocols can be used for transcriptomic analysis to increase the quantity of molecular samples for analysis, proteomics and metabolomics rely either upon more starting material for FACS or upon increased power detection and quantification in the analytical technologies. However, headway is being made and the feasibility is increasing of studying the metabolome (reviewed in Rubakhin et al., 2013), proteome (Taniguchi et al., 2010; Salehi-Reyhani et al., 2011), and epigenome (Kantlehner et al., 2011 ) in cell types, and the transcriptome in single cells (Hartmann and Klein, 2006; Hebenstreit, 2012). For example, Petricka et al., (2012) were able to obtain a dataset for approximately $10 \%$ of the proteome in the Arabidopsis root, by using stringent parameters for protein identification to ensure accuracy, whereas Moussaieff et al., (2013) used relative metabolomic data from wild-type whole roots and sorted cells (without fluorescent gating) to normalise data obtained from individual cell types. FACS has also been utilized to examine the distribution of the ubiquitous, but highly cell-specific small hormone molecule auxin (Petersson et al., 2009), combining cell sorting with mass spectrometry analysis. For each of these applications, FACS offers more than other cell-specific technologies because it is not restricted to delivering the active transcriptome of cells and allows a large number of similar cells to be harvested in rapid succession (Rogers et al., 2012).

Arecent direction in systems biology is the study of heterogeneity and stochasticity within cell types to gain a finer mechanistic level of detail on cell-specific responses (Munsky et al., 2012). By analysing gene expression in large numbers of individual cells cell-by-cell, it is possible to elucidate variability and the degree of noise within a cell type. The output from FACS can be isolated into plates of up to 384 wells in most machines, allowing for sufficient sampling of cell populations, whereas the amplification of mRNA from a single cell for microarray analysis is feasible by means of state-of-the-art RNA amplification kits. For instance, individual human epidermal cells were isolated and gene abundance measured both gene-bygene by polymerase chain reaction (PCR) optimised for single-cell analysis and genomically by Illumina BeadArray microarray (Tan 
et al., 2013). Hierarchical clustering demonstrated that epidermal cells had a relatively high preservation level of global gene expression profiles within subpopulations of cells in cell-to-cell replicates.

RNA sequencing methods have been developed for the small amounts of RNA obtainable from single cells (single cell RNAsequencing [scRNA-seq]; Hebenstreit, 2012). In mouse blastocysts, scRNA-seq has been used to investigate the transition of cells in the inner mass to embryonic stem cells, uncovering large transcriptomic changes and alternative splicing (Tang et al., 2010). Each of the four current methods for scRNA-seq still requires RNA amplification and, thus, introduction of potential amplification bias; however, the optimisation of on-FlowCell Reverse Transcription Sequencing (Mamanova and Turner, 2011) presents an attractive future for single-cell transcriptomics. Combined with FACS, this method will open up a new way to study plants on the cell-specific level.

\section{Conclusions and perspectives}

Accuracy and applications have greatly increased since details of the first FACS instrument were published. While the principles and mechanisms of more recent machines have remained similar, advances in molecular biology and the availability of transgenic lines have improved our ability to efficiently and accurately obtain subpopulations of single cell-types. FACS was originally used to isolate cells expressing a single fluorescent marker, but the combinations of lasers, detectors, and filters available on the current generation of cell sorters allow the observation of multiple fluorophores and four-way sorting.

It is a core goal of systems biology to be able to meaningfully link biological processes occurring in a cell with the functionality of entire organisms and even populations. Single-cell techniques bring measurements previously available only for large portions of the plant (such as leaf, root, or cell file), to a new frontier, bringing bottom-up and top-down research approaches closer together. At present, most work using FACS in plants has used Arabidopsis, but this is likely only due to the paucity of suitable transgenic fluorescent cell-type-specific marker lines in other species. This gap is already being narrowed, for instance for rice (Oryza sativa) (Evrard et al., 2012) and can be mitigated by employing other cell-type level methods (such as laser capture analysis of rice cell types; Jiao et al., 2009). Put together, it is likely that in the future a much greater share of 'omics' analysis across all species will occur at the level of the individual cell.

\section{Acknowledgements}

We thank Jo Hulsmans for helpful comments on the manuscript. This work was supported by a Biotechnology and Biological Sciences Research Council (BBSRC) new investigator (BB/H109502/1) grant to M.L.G., an Engineering and Physical Sciences Research Council/BBSRC-funded Warwick Systems Biology Doctoral Training Centre to A.C., and a University of Warwick Postgraduate Research Scholarship and a Stapley Educational Trust grant to R.B.

\section{References}

BARGMANN BOR, BIRNBAUM KD. (2009). Positive fluorescent selection permits precise, rapid, and indepth overexpression analysis in plant protoplasts. Plant Physiol 149: 1231-1239.

BECKER JD, BOAVIDA LC, CARNEIRO J, HAURY M, FEIJÓ JA. (2003). Transcriptional profiling of Arabidopsis tissues reveals the unique characteristics of the pollen transcriptome. Plant Physiol 133: 713-725.
BIRNBAUM K, JUNG JW, WANG JY, LAMBERT GM, HIRST JA, GALBRAITH DW BENFEY PN. (2005). Cell type-specific expression profiling in plants via cell sorting of protoplasts from fluorescent reporter lines. Nat Methods 2: 615-619.

BIRNBAUM K, SHASHA DE, WANG JY, JUNG JW, LAMBERT GM, GALBRAITH DW, BENFEY PN. (2003). A gene expression map of the Arabidopsis root. Science 302: 1956-1960.

BONNER WA, HULETT HR, SWEET RG, HERZENBERG LA. (1972). Fluorescence activated cell sorting. Rev Sci Instrum 43: 404-409.

BRADY SM, ORLANDO DA, LEE J-Y, WANG JY, KOCH J, DINNENY JR, MACE D, OHLER U, BENFEY PN. (2007). A high-resolution root spatiotemporal map reveals dominant expression patterns. Science 318: 801-806.

DAVIES WJ, WILKINSON S, LOVEYS B. (2002). Stomatal control by chemical signalling and the exploitation of this mechanism to increase water use efficiency in agriculture. New Phytol 153: 449-460.

DE SMETI, TETSUMURAT, DE RYBELB, FREY DIT FREIN, LAPLAZE L, CASIMIRO I, SWARUP R, NAUDTS M, VANNESTE S, AUDENAERT D, INZÉ D, BENNETT MJ, BEECKMAN T. (2007). Auxin-dependent regulation of lateral root positioning in the basal meristem of Arabidopsis. Development 134: 681-690.

DE SMET I, VASSILEVA V, DE RYBEL B, LEVESQUE MP, GRUNEWALD W, VAN DAMME D, VAN NOORDEN G, NAUDTS M, VAN ISTERDAEL G, DE CLERCQR, WANG JY, MEULI N, VANNESTE S, FRIML J, HILSON P, JÜRGENS G, INGRAM GC, INZÉ D, BENFEY PN, BEECKMAN T. (2008). Receptor-like kinase ACR4 restricts formative cell divisions in the Arabidopsis root. Science 322: 594-597.

DEALRB, HENIKOFF S. (2010). A simple method for gene expression and chromatin profiling of individual cell types within a tissue. Dev Cell 18: 1030-1040.

DINNENY JR, LONG TA, WANG JY, JUNG JW, MACE D, POINTER S, BARRON C, BRADY SM, SCHIEFELBEIN J, BENFEY PN. (2008). Cell identity mediates the response of Arabidopsis roots to abiotic stress. Science 320: 942-945.

EVRARDA, BARGMANNBOR, BIRNBAUM KD, TESTERM, BAUMANNU, JOHNSON AAT. (2012). Fluorescence-activated cell sorting for analysis of cell typespecific responses to salinity stress in Arabidopsis and rice. Methods MolBiol913:265-276.

FERRÁNDIZ C, GU Q, MARTIENSSEN R, YANOFSKY MF. (2000). Redundant regulation of meristem identity and plant architecture by FRUITFULL, APETALA1 and CAULIFLOWER. Development 127: 725-734.

GALBRAITHD. (2012). Flow cytometry and cell sorting: the next generation. Methods 57: $249-250$.

GEURTSR, FEDOROVAE, BISSELING T. (2005). Nod factor signaling genes and their function in the early stages of Rhizobium infection. Curr Opin Plant Biol8:346-352.

GIFFORD ML, DEAN S, INGRAM GC. (2003). The Arabidopsis ACR4 gene plays a role in cell layer organisation during ovule integument and sepal margin development. Development 130: 4249-4258.

GIFFORD ML, DEAN A, GUTIERREZ RA, CORUZZI GM, BIRNBAUM KD. (2008). Cell-specific nitrogen responses mediate developmental plasticity. Proc NatlAcad Sci USA 105: 803-808

GRØNLUND JT, EYRES A, KUMAR S, BUCHANANWOLLASTON V, GIFFORD ML. (2012). Cell specific analysis of Arabidopsis leaves using fluorescence activated cell sorting. $J$ Vis Exp 68: e4214.

HARTMANN CH, KLEIN CA. (2006). Gene expression profiling of single cells on large-scale oligonucleotide arrays. Nucleic Acids Res 34: e143.

HASELOFF J. (1998). GFP variants for multispectral imaging of living cells. Methods Cell Biol 58: 139-151.

HEBENSTREIT D. (2012). Methods, challenges and potentials of single cell RNAseq Biology 1: 658-667.

HIRSCH AM, KAPULNIK Y. (1998). Signal transduction pathways in mycorrhizal associations: comparisons with the Rhizobium-legume symbiosis. Fungal Genet Biol 23: 205-212.

JIAO Y, TAUSTA SL, GANDOTRA N, SUN N, LIU T, CLAY NK, CESERANI T, CHEN M, MA L, HOLFORD M, ZHANG H-Y, ZHAO H, DENG X-W, NELSON T. (2009). A transcriptome atlas of rice cell types uncovers cellular, functional and developmental hierarchies. Nat Genet 41: 258-263.

JULIUS MH, MASUDA T, HERZENBERG LA. (1972). Demonstration that antigenbinding cells are precursors of antibody-producing cells after purification with a fluorescence-activated cell sorter. Proc Natl Acad Sci USA 69: 1934-1938.

KANTLEHNER M, KIRCHNER R, HARTMANN P, ELLWART JW, ALUNNIFABBRONI M, SCHUMACHER A. (2011). A high-throughput DNA methylation analysis of a 


\section{A.D. Carter et al.}

single cell. Nucleic Acids Res 39: e44.

KIDNER C, SUNDARESAN V, ROBERTS K, DOLAN L. (2000). Clonal analysis of the Arabidopsis root confirms that position, not lineage, determines cell fate. Planta 211: 191-199.

KLEBER R, KEHR J. (2006). Preparation and quality assessment of RNA from cellspecific samples obtained by laser microdissection. Methods Mol Bio/323:367-377.

LAU OS, BERGMANN DC. (2012). Stomatal development: a plant's perspective on cell polarity, cell fate transitions and intercellular communication. Development 139: 3683-3692.

LAUX T, WÜRSCHUM T, BREUNINGER H. (2004). Genetic regulation of embryonic pattern formation. Plant Cell 16: S190-S202.

LIU J, LUOX, SHAFF J, LIANG C, JIAX, LIZ, MAGALHAES J, KOCHIAN LV. (2012). A promoter-swap strategy between the AtALMT and AtMATE genes increased Arabidopsis aluminum resistance and improved carbonuse efficiency for aluminum resistance. Plant J 71: 327-337.

MALAMY JE, BENFEY PN. (1997). Organization and cell differentiation in lateral roots of Arabidopsis thaliana. Development 124: 33-44.

MAMANOVA L, TURNER DJ. (2011). Lowbias, strandspecific transcriptome Illumina sequencing by on-flowcell reverse transcription (FRTseq). Nat Protoc6: 1736-1747.

MARTIN C, PAZARES J. (1997). MYB transcription factors in plants. Trends Genet 13: $67-73$.

MATTANOVICH D, BORTH N. (2006). Applications of cell sorting in biotechnology. Microb Cell Fact 5: 12.

MORISON JIL. (2006). Sensitivity of stomata and water use efficiency to high $\mathrm{CO}_{2}$. Plant Cell Environ 8: 467-474.

MOUSSAIEFFA, ROGACHEV I, BRODSKYL, MALITSKYS, TOALTW, BELCHERH, YATIV M, BRADYSM, BENFEY PN, AHARONIA. (2013). High-resolution metabolic mapping of cell types in plant roots. Proc Natl Acad Sci USA 110: E1232-E1241.

MUNSKY B, NEUERT G, VAN OUDENAARDEN A. (2012). Using gene expression noise to understand gene regulation. Science 336: 183-187.

MUSTROPH A, ZANETTI ME, JANG CJH, HOLTAN HE, REPETTI PP, GALBRAITH DW, GIRKE T, BAILEYSERRES J. (2009). Profiling translatomes of discrete cell populations resolves altered cellular priorities during hypoxia in Arabidopsis. Proc Natl Acad Sci USA 106: 18843-18848.

NAKAJIMA K, SENA G, NAWY T, BENFEY PN. (2001). Intercellular movement of the putative transcription factor SHR in root patterning. Nature 413: 307-311.

NAWYT, LEE J.Y, COLINAS J, WANG JY, THONGROD SC, MALAMY JE, BIRNBAUM K, BENFEY PN. (2005). Transcriptional profile of the Arabidopsis root quiescent center. Plant Cell 17: 1908-1925.
NELSON M. (1999). An overview of the use of flow cytometry in the analysis of mixed red cell populations. Pathology 31: 191-198.

OLDROYD GED, DOWNIE JA. (2008). Coordinating nodule morphogenesis with rhizobial infection in legumes. Annu Rev Plant Biol 59: 519-546.

PETERSSON SV, JOHANSSONAI, KOWALCZYKM, MAKOVEYCHUKA, WANG JY, MORITZT, GREBE M, BENFEY PN, SANDBERG G, LJUNG K. (2009). An auxin gradient and maximum in the Arabidopsis root apex shown by high-resolution cell-specific analysis of IAA distribution and synthesis. Plant Cell 21: 1659-1668.

PETRICKA JJ, SCHAUER MA, MEGRAW M, BREAKFIELD NW, THOMPSON JW, GEORGIEV S, SODERBLOM EJ, OHLER U, MOSELEY MA, GROSSNIKLAUS $\mathrm{U}$, BENFEY PN. (2012). The protein expression landscape of the Arabidopsis root. Proc Natl Acad Sci USA 109: 6811-6818.

R DEVELOPMENT CORE TEAM (2012). R: A language and environment for statistical computing. Vienna, Austria, R Foundation for Statistical Computing.

ROGERS ED, JACKSON T, MOUSSAIEFF A, AHARONI A, BENFEY PN. (2012). Cell typespecific transcriptional profiling: implications for metabolite profiling. Plant J 70: 5-17.

RUBAKHIN SS, LANNI EJ, SWEEDLER JV. (2013). Progress toward single cell metabolomics. Curr Opin Biotechnol 24: 95-104.

RUSHTON PJ, SOMSSICH IE, RINGLER P, SHEN QJ. (2010). WRKY transcription factors. Trends Plant Sci 15: 247-258.

SALEHIREYHANI A, KAPLINSKY J, BURGINE, NOVAKOVAM, DEMELLOAJ, TEMPLER RH, PARKER P, NEIL MAA, CES O, FRENCH P, WILLISON KR, KLUG D. (2011). A first step towards practical single cell proteomics: a microfluidic antibody capture chip with TIRF detection. Lab Chip 11: 1256-1261.

SCHOLL RL, MAY ST, WARE DH. (2000). Seed and molecular resources for Arabidopsis. Plant Physiol 124: 1477-1480.

TAN DWM, JENSEN KB, TROTTER MWB, CONNELLY JT, BROAD S, WATT FM. (2013). Single-cell gene expression profiling reveals functional heterogeneity of undifferentiated human epidermal cells. Development 140: 1433-1444.

TANG F, BARBACIORU C, BAO S, LEE C, NORDMAN E, WANG X, LAO K, SURAN MA. (2010). Tracing the derivation of embryonic stem cells from the inner cell mass by singlecell RNASeq analysis. Cell Stem Cell 6: 468-478.

TANIGUCHI Y, CHOI PJ, LI G.W, CHEN H, BABU M, HEARN J, EMILI A, XIE XS (2010). Quantifying E. coli proteome and transcriptome with single-molecule sensitivity in single cells. Science 329: 533-538.

YADAV RK, GIRKE T, PASALA S, XIE M, REDDY GV. (2009). Gene expression map of the Arabidopsis shoot apical meristem stem cell niche. Proc Natl Acad Sci USA 106: 4941-4946. 